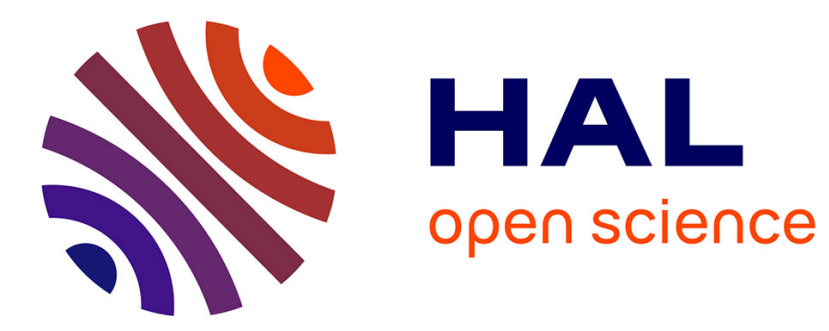

\title{
Chemins de fer et vision des paysages anglais \\ Charles-François Mathis
}

\section{To cite this version:}

Charles-François Mathis. Chemins de fer et vision des paysages anglais. Histoire, économie et société, 2005, 24 (1), pp.123-146. 10.3406/hes.2005.2539 . hal-02544838

\section{HAL Id: hal-02544838 \\ https://hal.science/hal-02544838}

Submitted on 16 Apr 2020

HAL is a multi-disciplinary open access archive for the deposit and dissemination of scientific research documents, whether they are published or not. The documents may come from teaching and research institutions in France or abroad, or from public or private research centers.
L'archive ouverte pluridisciplinaire HAL, est destinée au dépôt et à la diffusion de documents scientifiques de niveau recherche, publiés ou non, émanant des établissements d'enseignement et de recherche français ou étrangers, des laboratoires publics ou privés. 


\section{Chemins de fer et vision des paysages anglais}

Charles-François Mathis

\section{Citer ce document / Cite this document :}

Mathis Charles-François. Chemins de fer et vision des paysages anglais. In: Histoire, économie et société, $2005,24^{\mathrm{e}}$ année, $\mathrm{n}^{\circ} 1$. Cultures politiques, identités sociales en Grande-Bretagne. pp. 123-146;

doi : https://doi.org/10.3406/hes.2005.2539

https://www.persee.fr/doc/hes_0752-5702_2005_num_24_1_2539

Fichier pdf généré le 21/12/2018 


\title{
Résumé
}

L'introduction des chemins de fer en Angleterre dans le premier tiers du XIXe siècle provoque des bouleversements considérables, tant sur le visage offert par le pays, que sur le regard qui est porté sur lui. Une résistance à l'expérience du voyage en train, et particulièrement à la façon dont elle modifie la perception des paysages traversés, est notable. C'est que les chemins de fer remettent en effet en cause les modes de vision du paysage tels qu'ils ont été élaborés par les théories romantiques et pittoresques. Un véritable trauma du regard se produit alors, perceptible dans les nombreux témoignages de l'époque, qui dénoncent un paysage fragmenté, confus, monotone, et au bout du compte trop détaché de celui qui voudrait l'admirer. D'où, sans doute, le reproche de déracinement que ses détracteurs opposent au chemin de fer, qui semble en effet mettre à mal les fondements d'une certaine définition de l'identité anglaise.

\begin{abstract}
When railways were introduced in England, they not only changed the face of the country, but also the ways it was perceived. A great number of contemporaries disliked the new perception of the landscapes offered by the experience of railway journeys, which seemed indeed to shatter the principles of this vision as they were established by picturesque and romantic theories and practices. Railways were thus blamed for offering fragmented, monotonous, whirling landscapes, and for creating a gap between the observer and the object of its admiration. Hence the criticism made by the fiercest opponents of railway travel, who denounced its destruction of a real knowledge of the country by its inhabitants, thus damaging a particular definition of English national identity.
\end{abstract}




\title{
Chemins de fer et vision des paysages anglais
}

\author{
par Charles-François MATHIS
}

\section{Résumé}

L'introduction des chemins de fer en Angleterre dans le premier tiers du XIX' siècle provoque des bouleversements considérables, tant sur le visage offert par le pays, que sur le regard qui est porté sur lui. Une résistance à l'expérience du voyage en train, et particulièrement à la façon dont elle modifie la perception des paysages traversés, est notable. C'est que les chemins de fer remettent en effet en cause les modes de vision du paysage tels qu'ils ont été élaborés par les théories romantiques et pittoresques. Un véritable trauma du regard se produit alors, perceptible dans les nombreux témoignages de l'époque, qui dénoncent un paysage fragmenté, confus, monotone, et au bout du compte trop détaché de celui qui voudrait l'admirer. D'où, sans doute, le reproche de déracinement que ses détracteurs opposent au chemin de fer, qui semble en effet mettre à mal les fondements d'une certaine définition de l'identité anglaise.

\section{Abstract}

When railways were introduced in England, they not only changed the face of the country, but also the ways it was perceived. A great number of contemporaries disliked the new perception of the landscapes offered by the experience of railway journeys, which seemed indeed to shatter the principles of this vision as they were established by picturesque and romantic theories and practices. Railways were thus blamed for offering fragmented, monotonous, whirling landscapes, and for creating a gap between the observer and the object of its admiration. Hence the criticism made by the fiercest opponents of railway travel, who denounced its destruction of a real knowledge of the country by its inhabitants, thus damaging a particular definition of English national identity.

Au cours de la plupart des voyages en train [...], le visage de la nature, les belles perspectives de collines et vallées, sont perdus ou déformés au regard. L'alternance de monts et de vaux, l'air sain, et toutes ces associations grisantes liées à «la Route», sont perdus ou transformés en mornes tranchées, tunnels lugubres, et effluves nocives de la locomotive hurlante. ${ }^{1}$

1. «In travelling on most of the railways [...], the face of nature, the beautiful prospects of hill and dale, are lost or distorted to our view. The alternation of high and low ground, the healthful breeze, and all those exhilarating associations connected with "the Road", are lost or changed to doleful cuttings, dismal tunnels, and the noxious effluvia of the screaming engine. " Anonyme, Horse-Power Applied to Railways at Higher Rates of Speed than by Ordinary Draught, London, 1844, p. 48. 
Le sentiment de perte qui s'exprime dans cet extrait se retrouve chez la plupart des Victoriens ayant partagé leur expérience d'un voyage en train. Presque tous font état de cette vue déformée de la nature, de ces paysages qu'ils ont l'impression de traverser sans les voir. Ces exclamations, portant en fin de compte sur la vitesse excessive des chemins de fer, peuvent paraître aujourd'hui étonnamment exagérées, et il est difficile de croire que les quelques 40 à 50 miles en pointe que les trains victoriens atteignaient au mieux au milieu du siècle ${ }^{2}$ aient pu poser un obstacle physiologique réel à la vision des paysages se déroulant sous leurs yeux. Cette vitesse, qui semble dérisoire de nos jours, représentait pourtant un bouleversement profond pour un monde habitué aux déplacements cahoteux, pittoresques, et relativement lents des diligences.

C'est en 1830 que le chemin de fer a fait son entrée fracassante dans le monde anglais. Certes, dès 1825 était ouverte la ligne Stockton-Darlington au nord-est du pays, mais il ne s'agissait encore que d'une ligne utilisée essentiellement à des fins industrielles (le transport du charbon). Il faut attendre les débats violents au Parlement et dans la presse à propos de la Manchester and Liverpool Railway Bill, la fameuse compétition de Rainhill qui vit la victoire de la locomotive Rocket de Stephenson sur ses adversaires, et surtout l'ouverture très médiatisée de la ligne Manchester-Liverpool (la première ligne commerciale pour le transport de passagers) le 15 septembre 1830 , pour que le train s'impose vraiment sur la scène publique. Malgré des augures sombres (la mort de William Huskisson, écrasé par un train le jour de l'inauguration), le succès fut immédiat $(400000$ spectateurs se seraient déplacés pour assister à l'événement), et les années 1830 et 1840 furent ponctuées de booms économiques liés aux spéculations ferroviaires, en 1836-1837 notamment, et plus dramatiquement en 18461849 , cette dernière période s'achevant par un effondrement boursier d'une rare violence. D'un réseau de moins de 100 miles en 1830 , on passe à quelques 6000 miles en 1850 , et les presque six millions de visiteurs de l'Exposition universelle du Crystal Palace en 1851 n'auraient jamais pu être atteints sans les facilités de déplacement offertes par la révolution ferroviaire.

Si ce nouveau mode de transport acquiert rapidement la faveur du public, les interrogations sont fortes dans les vingt premières années de son développement sur ses conséquences sur la société victorienne. Bien vite devenu le symbole même des transformations industrielles du pays, le chemin de fer doit subir des attaques venues de tous les horizons et portant sur tous ses aspects, de la démocratisation du transport qu'il permettait ${ }^{3}$, aux nuisances que son passage provoquait aux abords des parcs de la noblesse ou des demeures de simples particuliers ${ }^{4}$, en passant par ses supposées atteintes à la santé des passagers ${ }^{5}$ (combien de batailles médicales pour déterminer des effets

2. En 1845, la vitesse moyenne la plus élevée atteinte par les trains commerciaux était de 43 miles par heure sur la Great Western Line.

3. C'est, au fond, le sens des craintes de Wordsworth à propos de la ligne Kendal-Windermere à laquelle il s'oppose en 1844-1845.

4. «On the very line of this railway, I have built a comfortable house; it enjoys a pleasing view of the country. Now judge, my friend, of my mortification, whilst I am sitting comfortably at breakfast with my family, enjoying the purity of the summer air, in a moment my dwelling [...] is filled with dense smoke of foetid gas; my homely, though cleanly, table covered with dirt; and the features of my wife and family almost obscured by a polluted atmosphere.» «Sur la ligne même du chemin de fer, $\mathrm{j}$ 'ai fait construire une maison confortable, qui offre une belle vue sur la campagne. Jugez, mon ami, de ma mortification, lorsqu'en un moment, alors que je prends confortablement mon petit-déjeuner avec ma famille, mon habitation est emplie d'une fumée dense de gaz fétides; ma table simple et propre recouverte de saleté; et les traits de ma femme et de ma famille presque cachés par l'atmosphère polluée.» Lettre de «Ebenezer» au Leeds Intelligencer, 13 janvier 1825, p. 3.

5. Voir Arthur Freeling, The London and Birmingham Railway Companion, London, Whittaker, 1838, p. 33-36, où il est question des dangers (selon lui imaginaires) des tunnels. 
physiologiques de la vitesse, de la fumée, ou des secousses des wagons!). Parmi ces reproches et ces craintes, se développe toute une littérature essentiellement préoccupée de son impact sur la vision du paysage, dont l'extrait anonyme déjà cité donne un bon aperçu.

Le malaise qui s'en dégage semble bien avoir un fondement culturel. Certes, la nouveauté de ce mode de transport imposait un apprentissage du regard, mais le simple fait que cette adaptation ait été pour certains douloureuse, impossible, ou ressentie comme une perte, souligne assez qu'elle allait à l'encontre de données profondément ancrées dans les mentalités de la première moitié du XIX ${ }^{e}$ siècle. Quel est dès lors ce substrat que le chemin de fer mettait en cause, quels sont précisément les bouleversements qu'il a produit dans l'attitude des Victoriens face aux paysages anglais, et comment y ont-ils répondu?

Wolfgang Schivelbusch, dans sa remarquable étude sur le voyage en train en Europe ${ }^{6}$, a déjà donné les premiers éléments de réponse à ces interrogations. Il souligne en effet à quel point le train, en détruisant la relation directe qui existait auparavant entre l'espace traversé et le voyageur (le premier plan de la vue, qui était celui de cette interaction et de l'expérience quotidienne du voyageur avant la révolution ferroviaire, est effacé par la vitesse, obligeant à porter le regard sur les choses distantes), rend difficile pour ce dernier de (re)connaître le paysage qu'il parcourt. Cette coupure entre le voyageur et ce qui se déroule devant lui est au cœur même du sentiment de manque évoqué par les contemporains, qui se retrouvent face à ce que Schivelbusch appelle un paysage panoramique. Il reste à comprendre à quoi se heurtait cette perception nouvelle, et pourquoi elle a provoqué, dans le cadre précis de l'expérience anglaise, de si vifs débats, mettant en jeu, au bout du compte, la définition même de l'identité nationale.

\section{Les racines pittoresques et romantiques de la vision du paysage}

Pour comprendre les raisons de la résistance culturelle d'une partie de la population anglaise à l'expérience visuelle offerte par les chemins de fer, il convient de revenir à la source de l'intérêt pour les paysages qui se situe, en Angleterre, dans la deuxième moitié du XVIII ${ }^{e}$ siècle avec le développement de la pensée pittoresque. Pour qu'un espace naturel puisse être considéré comme un paysage, il est impératif qu'un certain nombre de conditions culturelles soient réunies, ce qui ne devient le cas outre-Manche qu'avec l'admiration progressive pour les ouvres de Claude et de Salvatore Rosa ${ }^{7}$, la mode du Grand Tour en Italie, et le retour de Wilson de Rome qui marque pour certains le début de la peinture de paysage anglaise. C'est par ces derniers que les Anglais ont appris à regarder esthétiquement un paysage, et les jardins et parcs de «Capability» Brown doivent se comprendre comme une succession de scènes, de vues découvertes au détour de chemins sinueux, faites de bosquets, de pelouses impeccables, de rivières tortueuses. Il est important de bien comprendre la source picturale de l'appréciation des paysages anglais, car son influence se fait sentir au moins jusqu'au milieu du $\mathrm{XIX}^{\mathrm{e}}$ siècle. Le terme landscape lui-même, comme l'a bien montré John Barrell ${ }^{8}$, a d'abord désigné une peinture de paysage, avant de s'appliquer à un lieu

6. Wolfgang Schivelbusch, The Railway Journey: the Industrialization of Time and Space in the XIXth century, Leamington Spa, Berg, 1986 (1 ${ }^{\text {re }}$ ed. allemande 1977).

7. Paul Shepard, Man in the Landscape: A Historic View of the Esthetics of Nature, College Station (Texas), A\&M University Press, 1991 (1 $1^{\text {re }}$ é.1967)

8. John Barrell, The Idea of Landscape and the Sense of Place 1730-1840: An Approach to the Poetry of John Clare, Cambridge, Cambridge University Press, 1972. 
précis regardé d'un certain point de vue, pour finalement s'appliquer plus généralement à l'ensemble des espaces naturels, devenant tous sujets potentiels d'une approche picturale. Apprendre à regarder un paysage devient une pratique commune à l'ensemble des classes supérieures de la fin du XVII' ${ }^{c}$ siècle, un réquisit culturel indispensable. Et ce regard se fait selon l'expérience transmise par les peintures de Claude et Rosa, en insistant sur la composition du paysage: la recherche du point de vue juste ou la sélection des éléments à inclure (Gilpin est resté fameux pour ses choix qui, au bout du compte, empêchaient de reconnaître l'endroit qu'il représentait...). Célèbre est ainsi la scène de Northanger Abbey de Jane Austen où Henry Tilney éduque la vision de Catherine pour l'ouvrir à la beauté pittoresque, et plus particulièrement la condamnation finale, par la disciple zélée, d'une vue de Bath. On a là l'essence même de ce que William Gilpin théorise dans ses différents récits de voyages (en particulier ses célèbres Observations on the River Wye publiées en $1782^{\circ}$ ) comme le pittoresque, à savoir «that kind of beauty which would look well in a picture» ${ }^{10}$. Cette façon de voir devient tellement populaire, notamment dans les années 1790, qu'elle façonne pour des décennies le regard porté par les classes moyennes et supérieures sur les paysages anglais. Au prix de la précision et des nuances développées par les penseurs du pittoresque, William Gilpin et Uvedale Price en tête. De ce dernier, qui publie ses deux volumes d'Essay on the Picturesque entre 1794 et $1798^{~}{ }^{11}$, on retient essentiellement son insistance sur la variété des scènes représentées. «Price replace la source du pittoresque dans les détails irréguliers, les surfaces rugueuses, et les textures grossières de la nature et de l'art, qui flattent l'oil par leur clair-obscur.» ${ }^{12}$ L'accent est mis sur l'irrégularité, les bosquets, ruines, arbres tordus, rivières sinueuses qui brisent la monotonie d'une vue. S'adressant aussi aux concepteurs de parcs, Price s'inscrit toujours dans l'idée d'une nécessaire construction du paysage, ne serait-ce que par la quête du point de vue juste.

Dans la vulgarisation de la théorie pittoresque, la particularité de chaque paysage mise en avant par William Gilpin ou Richard Payne Knight vient à se perdre dans l'uniformité des regards portés sur elle ${ }^{13}$. Le voyage dans le domaine des Lacs devient ainsi un impératif pour tout touriste de la fin du XVIII siècle et du début du XIX ${ }^{e}$ siècle. On y cherche les vues suggérées par Thomas West dans son guide publié en 1778 , et tellement populaire qu'il en est à sa dixième édition en $1812^{14}$. Le pittoresque à ce moment là est devenu une convention et les façons de voir le paysage totalement formalisées ${ }^{15}$. C'est notamment contre cela que vient s'élever le romantisme, sans pour autant parvenir à bouleverser complètement des habitudes visuelles déjà bien ancrées

9. William Gilpin, Observations on the River Wye and Several Parts of South Wales, \& Relative Chiefly to Picturesque Beauty; Made in the Summer of the Year 1770, London, 1782.

10. William Gilpin, Essay Upon Prints, London, Robson, 1768, p. 2.

11. Uvedale Price, An essay on the Picturesque as compared with the Sublime and the Beautiful: and on the use of studying pictures for the purpose of improving real landscape, 2 vol., London, Hereford, 1794-1798.

12. «Price redefined the source of the picturesque as those irregular details, rough surfaces, and coarse textures in nature and art that pleased the eye with their shadowy chiaroscuro.» Paul Shepard, op. cit., p. 126.

13. Ann Bermingham, Landscape and Ideology: The English Rustic Tradition 1740-1860, London, Thames and Hudson, 1987 (1 $1^{\text {rc }}$ é. 1986). 1778.

14. Thomas West, A Guide to the Lakes [...] in Cumberland, Westmorland and Lancashire, London,

15. Esther Moir, The Discovery of Britain: The English Tourists, London, Routledge and Kegan, 1964. 
dans les mentalités. Wordsworth, en particulier, cherche à redonner vie à l'esprit de chaque endroit, en insistant sur le lien émotionnel, spirituel même parfois, qui peut s'établir entre le voyageur, qui dans l'idéal n'est plus seulement un spectateur mais un participant au lieu, et le paysage qu'il découvre. L'attention au détail - déjà en germe dans certaines versions du pittoresque - est plus poussée (et Wordsworth, comme la plupart de ses contemporains, avait une bonne connaissance de l'histoire naturelle), et l'accent est mis sur l'expérience vécue du lieu. L'expérience du «Wanderer» de The Excursion est celle d'une vision juste de la nature - Ruskin dira sa dette à Wordsworth dans son apprentissage du regard -, et d'un contact direct avec l'ordre naturel.

C'est bien de cela dont il est question dans son fameux Guide to the Lakes dès sa première édition. Un guide qui s'adresse non seulement au touriste de passage, mais aussi à ceux qui cherchent à vivre dans ce lieu-phare de la beauté naturelle anglaise. Wordsworth y manifeste son désir de libérer le touriste des conventions imposées à son regard: «Admettant l'arrivée inévitable d'étrangers à la région, Wordsworth espérait les tirer hors du cadre accepté de leur expérience des Lacs, et leur montrer la campagne libérée (relativement) des clichés, les mettant en demeure d'y répondre avec imagination, sans entraves. ${ }^{16}$

Les différentes éditions de ce guide, de la première en 1810 entièrement ancrée dans la tradition pittoresque et présentée comme une introduction littéraire aux Select Views in Cumberland, Westmoreland, and Lancashire, par le Révérend Joseph Wilkinson ${ }^{17}$, jusqu'à la cinquième et dernière édition de 1835 , qui forme un tout indépendant ${ }^{18}$, manifestent donc une forme d'affirmation de la pensée romantique hors du cadre étroit des théories pittoresques, mais ne parviennent pas à se détacher complètement de ces dernières. Wordsworth conseille toujours d'ignorer certains chemins qui ne donnent pas le bon point de vue sur tel ou tel paysage. Il insiste aussi sur la variété de la région des Lacs et de chacune de ses scènes, qui en fait le charme: le domaine des Lacs serait pour cette raison supérieur aux Alpes même. Quant aux endroits qu'il préfère, ils ont tout du credo pittoresque, avec leurs ondulations, irrégularités, arbres et rivières savamment agencés par la nature pour le plaisir des yeux, etc. Si l'expérience du paysage est moins superficielle et stéréotypée que celle dont avait finalement accouché la version vulgarisée du pittoresque, elle reste liée à cette dernière par bien des attaches.

Jusqu'aux années 1830 , donc, le regard anglais sur les paysages s'organise autour de ces divers éléments: recherche $d$ 'un point de vue précis qui donne la vue la plus pittoresque (la plus picturale) sur un lieu, ce qui implique une liberté de mouvement et de choix des éléments entrant dans la composition du paysage; attention au détail de la scène représentée sous les yeux, et participation concrète, personnelle, émotionnelle même, au paysage traversé; appréciation esthétique, enfin, accordée avant tout aux paysages faisant preuve de variété. Or ce sont justement ces points qui sont les uns après les autres réduits à rien ou bouleversés par l'irruption du chemin de fer, provoquant dès lors une résistance culturelle forte de la part de bien des voyageurs victo-

16. «Recognizing that outsiders would come, Wordswoth hoped to force them out of the accepted frames of reference for their experience of the Lakes and to show them the countryside stripped (relatively) free of cliché, calling upon them to answer with unfettered imaginations. "James Buzard, The Beaten Track: European Tourism, Literature and the Ways to "Culture" 1800-1918, Oxford, Clarendon Press, 1993, p. 30.

17. William Wordsworth, Scenery of the Lakes, dans Rev. Joseph Wilkinson, Select Views in Cumberland, Westmoreland, and Lancashire, London, Ackermann, 1810.

18. William Wordsworth, A Guide Through the District of the Lakes in The North of England, with A Description of the Scenery, etc. For the Use of Tourists and Residents, Kendal, Hudson and Nicholson I London, Longman \& Co., Moxon, and Whittaker \& Co., 1835. 
riens à une expérience visuelle proprement révolutionnaire, et face à laquelle ils étaient conceptuellement démunis.

\section{$L$ 'expérience du voyage en train}

Les témoignages de contemporains narrant leurs voyages en train sont nombreux, et permettent d'apprécier la nature de leurs critiques concernant les scènes qui défilaient sous leurs yeux. La fascination d'une telle expérience est toujours évoquée, mais elle charrie souvent avec elle des reproches parfois vifs.

Le premier d'entre eux, le plus évident, concerne la possibilité matérielle de voir le paysage. Les chemins de fer, ne s'embarrassant guère d'obstacles, traversent sans sourciller montagnes et précipices, fleuves et vallées. Le voyageur subit ainsi une succession de tunnels, de tranchées, de talus, de viaducs, qui tous modifient sa façon d'appréhender le paysage, soit qu'ils oblitèrent ce dernier, soit qu'ils le surplombent. $\mathrm{La}$ vue du train devient ainsi partielle, discontinue, et provoque de ce fait la frustration des critiques du chemin de fer. Même les commentateurs les plus obligeants déplorent parfois cet obstacle à leur contentement visuel. Comme le dit justement l'auteur des guides Drake sur le trajet Birmingham-Liverpool, «Bostock Hall et Wharton seraient vus à l'est de la ligne après avoir quitté la gare, si le train n'entrait dans une tranchée peu profonde, dont les bords cachent la vue "au moment précis où il ne le faudrait pas".» ${ }^{19}$ Quant à Frederick Law Olmsted, très pressé de voir la campagne anglaise lors d'un voyage qu'il effectue en mai 1850 à partir de Birkenhead:

Un lourd nuage assombrissait le paysage, et alors que nous émergions d'un tunnel obscur, en tourbillonnant hors de la ville, de grosses gouttes de pluie vinrent s'abattre obliquement sur nous. Une femme toussa, et nous fermâmes la fenêtre. La route traversait une tranchée profonde, et seuls des abaissements occasionnels de son talus verdi de pelouse permettaient, à travers l'herbe poussiéreuse, quelques aperçus fugitifs de la campagne. En lueurs successives:

Un potager, avec des rangées de jeunes choux, de laitues et de pois;

Au dessus d'une haie, une jolie villa en pierres neuves, où le jardinier claquait les fenêtres de la serre et les femmes de chambre arrachaient les habits des cordes à linge;

Un pont, avec des enfants criant et agitant leurs chapeaux;

Un champ de ble, aux sillons tellement droits, et à la terre si propre et bien labourée, qu'on aurait dit une plante de jardin;

Un bout d'un vaste pâturage, où des poulains et des vaches tournaient le dos aux bourrasques de pluie; de longues collines dans le fond, avec quelques arbres et un clocher s'élevant derrière eux;

Quelques minutes encore de talus vert;

Une secousse - un arrêt. ${ }^{20}$

19. «Bostock Hall and Wharton would be seen $E$. from the railroad after leaving the station, but that the line enters a shallow cutting; the banks of which hide the view "just at the very time when they should not".» Drake's Road Book of the Grand Junction Railway, Birmingham, James Drake, n.d., p. 46.

20. "A heavy cloud darkened the landscape, and as we emerged in a few moments from a dark tunnel, whirling out of the town, big drops of rain came slanting in upon us. A lady coughed, and we closed the window. The road ran through a deep cutting, with only occasionally such depressions of its green-sodded bank, that we could, through the dusty glass, get gtimpses of the country. In successive gleams: A marketgarden, with rows of early cabbages, and lettuce, and peas; Over a hedge, a nice new stone villa, with the gardener shoving up the sashes of the conservatory, and the maids tearing clothes from the drying-lines; $A$ bridge, with children shouting and waving hats; $A$ field of wheat, in drills as precisely straight, and in earth as clean and finely-tilled, as if it were a garden-plant; $A$ bit of broad pasture, with colts and cows turning tail to the squall; long hills in the back, with some trees and a steeple rising beyond them; Another few minutes of green bank; A jerk - a stop.» Frederick Law Olmsted, Walks and Talks of an American Farmer in England, 1852, dans Humphrey Jennings, Pandaemonium: The coming of the machine as seen by contemporary observers, London, Picador (Pan Books), 1987, p. 259. 
Cet extrait rend bien compte de la vision nouvelle offerte par le train, faite d'instantanés (le parallèle avec la photographie qui fait alors ses premiers pas est frappant, ne serait-ce que dans l'image des lueurs successives que les talus laissent pénétrer dans l'œil du voyageur, et où s'impriment les images saisies de la campagne). Le procédé employé par Law Olmsted pour rendre compte de son expérience, l'énumération, dans un style qu'on pourrait appeler justement télégraphique (une autre invention dont l'essor est contemporain du chemin de fer, et largement favorisé par ce dernier...), est tout à fait représentatif de la plupart des autres témoignages de l'époque.

Ce découpage visuel de l'espace traversé est quelquefois poussé jusqu'à la limite des capacités de compréhension des voyageurs. En effet, la disposition physique des lignes de chemin de fer (passant dans des tranchées profondes au pied de gigantesques talus, surplombant les maisons des banlieues, traversant des vallées à des hauteurs insoupçonnées avant de plonger dans les tunnels), couplée à leur vitesse, a parfois tendance à produire une perte des repères spatiaux. L'ordre naturel est bouleversé au point d'égarer les sens du passager. Thomas Carlyle l'a évoqué dans une lettre écrite le 13 septembre 1839 à propos d'un voyage qu'il a effectué de nuit entre Carlisle et Londres:

Une pluie fine avait commencé entre temps; $j$ 'en sentis le premier crachin sur mon visage alors que nous roulions vers notre auberge à Preston. Tourbillonner dans cette obscurité confuse sur ces ailes de vapeur est l'une des choses les plus étranges que j'ai jamais vécues. Sifflant et se ruant en avant, sans qu'on sache vers où, on pouvait voir au loin la lueur des villes, des villes inconnues; nous passions au dessus des villes (je vis clairement une ville ou un village dont les cheminées se tendaient vainement vers nous); sous les étoiles, non sous les nuages mais parmi eux; hors d'un véhicule puis dans un autre, grognant, rugissant nous volions; - rien de plus comparable au vol de Faust sur le manteau du Diable; un peu comme si quelque énorme oiseau de nuit à vapeur vous avait jeté sur son dos, et glissait rapidement avec vous à travers l'espace inconnu, probablement vers Londres ! ${ }^{21}$

La nuit joue son rôle, certes, dans cette sensation d'égarement, mais le mouvement et le trajet mêmes du chemin de fer y contribuent fortement. L'allusion au pacte faustien n'est bien entendu pas anodine, en ce qu'elle accrédite l'idée d'une avancée contrenature: il y a quelque diablerie sans doute dans cette puissance redoutable qui oblitère toute notion de l'espace traversé pour emmener le voyageur, par des voies inconnues, vers une destination supposée!

Le vocabulaire utilisé ici par Carlyle est aussi révélateur de l'expérience des premiers passagers, en particulier l'emploi des verbes «tourbillonner» (to whirl) et «voler» (to fly). Ce sont les verbes que l'on retrouve dans presque chaque description de voyage en train. La perte de netteté de la vision des paysages y est palpable. Plus précisément, deux critiques sont sous-jacentes dans l'emploi de ces mots: dans le tourbillon qu'est devenu le paysage traversé, les éléments se mêlent dans la confusion, ils perdent de leur précision et de leur individualité; parallèlement, et presque a contrario,

21. «A small rain had begun by this time; the first spit of it I felt on my face as we drove up to our inndoor at Preston. The whirl thro' the confused darkness on those steam wings was one of the strangest things I have experienced. Hissing and dashing on, one knew not whither, we saw the gleam of towns in the distance, unknown towns; we went over the tops of towns (one town or village I saw clearly with its chimney heads vainly stretching up towards us); under the stars, not under the clouds but among them; out of one vehicle then into another, snorting, roaring we flew; - the likest thing to a Faust's flight on the Devil's mantle; or as if some huge steam nightbird had flung you on its back, and were [p. 183:J sweeping thro' unknown space with you, most probably towards London! » Thomas Carlyle, The Collected Letters of Thomas and Jane Welsh Carlyle, C.R. Sanders (ed.), Durham (North Carolina), Duke University Press, 1985, vol. 11 (1839), p. 182-183. 
les quelques objets qui se détachent de la masse sont perçus hors de leur contexte, sans attache, sans lien les uns avec les autres. Le paysage devient à la fois confus et fragmenté. C'est l'avènement de ce que Erwin Straus a appelé l'espace géographique, fait de changements brutaux provoquant une perte des repères intermédiaires, comme le fait remarquer justement Wolfgang Schivelbusch ${ }^{22}$. Une telle sensation est parfaitement perceptible dans la fameuse description faite par Dickens du voyage de Dombey après la mort de son fils:

Il ne trouvait dans la route ni plaisir ni distraction. Torturé par ces pensées, il emportait avec lui sa tristesse, au milieu de ces tableaux variés qui fuyaient devant lui, et, dans cette course rapide, qu'il faisait sans y songer, il ne voyait ni les sites pittoresques, ni les riches campagnes, mais toujours un pêle-mêle désolant de projets brisés et de pensées jalouses. La rapidité même du train semblait une raillerie cruelle de la course rapide de la vie que le jeune enfant avait passée sur la terre, entraîné par une puissance implacable vers le but fatal. [...] Et le convoi fuyait de la ville, sifflant, grondant, mugissant, s'enfonçant sous les demeures des hommes et faisant trembler les rues, serpentant un instant dans la plaine, disparaissant aussitôt dans les profondeurs de la terre, rugissant au milieu d'épaisses ténèbres, puis s'élançant de ce gouffre pour courir à la lumière et poursuivre sa marche rapide sous les rayons brillants du jour; oui, il fuyait toujours sifflant, grondant, mugissant à travers les champs, les bois, les moissons, les prairies, le sable, la terre, l'argile, le roc, rasant mille objets que le voyageur croit saisir et qui déjà sont bien loin de lui, et s'avançant entouré d'un horizon trompeur qui, malgré sa fuite rapide, marche lentement à ses côtés. Ne dirait-on pas la chasse inexorable de ce monstre au cœur de fer, la mort?

Oui, à travers les vallées, sur les hauteurs, au milieu des bruyères, des vergers, franchissant les parcs, les jardins, les canaux, les rivières, devant les troupeaux qui paissent, les moulins qui tournent, la barque qui flotte, les morts qui reposent, les fabriques qui fument, près du torrent qui bouillonne, des hameaux qui se groupent, de la haute cathédrale qui s'isole dans les airs, près des froids marécages, où la brise et l'ouragan soufflent à leur gré, le convoi passe et fuit sifflant, grondant, mugissant, ne laissant de son passage d'autre trace qu'un peu de poussière et un peu de fumée; ne dirait-on pas la chasse inexorable de ce monstre au cour de fer: la mort ? 23

On a là une accumulation qui semble briser justement la particularité de ce qui est parcouru, donnant le sentiment d'une confusion totale. Les objets aperçus s'enchaînent tellement vite les uns après les autres qu'il est quasiment impossible de les distinguer

22. Erwin Strauss, The Primary World of the Senses, New York/London, 1963, p. 319, cite par Wolfgang Schivelbusch, op. cit., p. 53.

23. «He found no pleasure or relief in the journey. Tortured by these thoughts he carried monotony with him, through the rushing landscape, and hurried headlong, not through a rich and varied country, but a wilderness of blighted plans and gnawing jealousies. The very speed at which the train was whirled along mocked the swift course of the young life that had been borne away so steadily and inexorably to its foredoomed end. [...] Away, with a shriek, and a roar, and a rattle, from the town, burrowing among the dwellings of men and making the streets hum, flashing out into the meadows for a moment, mining in through the damp earth, booming on in darkness and heavy air, bursting out again into the sunny day so bright and wide; away, with a shriek, and a roar, and a rattle, through the fields, through the woods, through the corn, through the hay, through the chalk, through the mould, through the clay, through the rock, among objects close at hand and almost within grasp, ever flying from the traveller, and a deceitful distance ever moving slowly within him: like as in the track of the remorseless monster, Death! Through the hollow, in the height, by the heath, by the orchard, by the park, by the garden, over the canal, across the river, where the sheep are feeding, where the mill is going, where the barge is floating, where the dead are lying, were the factory is smoking, where the stream is running, where the village clusters, where the great Cathedral rises, where the bleak moor lies, and the wild breeze smooths [sic] or ruffles at its inconstant will; away, with a shriek, and a roar, and a rattle, and no trace to leave behind but dust and vapour: like as in the track of the remorseless monster, Death! " Charles Dickens, Dombey and Son, 1846-1847, dans Humphrey, Jennings op. cit., p. 233-235 (traduction de P. Lorain, Dombey et Fils, Paris, Hachette, 1857 , vol. II, p. 314-315). 
entre eux, ou de les replacer dans un environnement précis. L'espace traversé par le train est ici en quelque sorte toute l'Angleterre (bien qu'on connaisse le trajet effectué, de Leamington à Birmingham, Dickens évoque aussi bien des paysages de landes, que des vergers, des bois, des pâturages ou des lieux industriels), et de ce fait ne peut être rattaché à aucun paysage particulier, à aucune région précise. Par ailleurs, dans cette énumération, les transitions sont absentes, le train les interdit. Ce n'est plus un pays unifié où l'on passe d'un paysage à un autre avec gradation et mesure, mais une succession d'éléments séparés.

Il est assez révélateur de comparer ce texte de Dickens avec sa description d'un voyage en diligence dans Martin Chuzzlewit:

Hue dia! elle plante là haies, grilles et arbres; chaumières, granges et travailleurs qui rentrent chez eux; hue dia! elle plante là les voitures à ânes garées dans le fossé, les carrioles vides aux chevaux déchaînés qui, stimulés du fouet, franchissent d'un bond le petit cours d'eau et que les charretiers doivent maintenir à grand-peine près de la barrière à cinq traverses jusqu'à ce que la diligence ait franchi le virage étroit de la route. Hue dia! elle plante là les églises abandonnées, seules dans leur coin tranquille, au milieu de leur cimetière rustique où les tombes sont vertes et où les pâquerettes dorment - car le soir tombe - sur le sein des morts. Hue dia ! elle plante là les ruisseaux où les bêtes se rafraîchissent les pattes et où poussent les joncs; les clôtures de parcs à chevaux, les fermes et les paillers, et les meules de l'année passée, entamées, tranche après tranche, et qui, dans la lumière déclinante, prennent l'allure de pignons en ruines, bruns et anciens. Hue dia! elle dévale la descente caillouteuse, passe à gué dans le joyeux éclaboussement et remonte au petit galop jusqu'à la route plane. Hue dia! Hue dia! ${ }^{24}$

Le mode littéraire est le même: répétition d'une formule, suivie d'éléments énumérés et accumulés. Pourtant la sensation qui se dégage de cet autre extrait n'a rien de l'angoisse et de l'oppression du premier, et cela n'est pas dû seulement aux circonstances émotionnelles bien différentes dans lesquelles sont effectués ces voyages. A mieux y regarder on se rend compte en effet qu'ici les objets évoqués le sont de manière plus précise (les adjectifs sont nombreux par exemple); on a accès au détail de chacun d'eux, et Dickens prend son temps pour les évoquer. Ils ne forment par ailleurs qu'un seul et même paysage qu'il est possible de reconstruire (la campagne anglaise, l'église rurale et son cimetière attenant, le cours d'eau qui passe non loin et les champs et les prés dans la lumière déclinante du soir), et non une succession informe d'endroits séparés. Les choses aperçues ne filent pas sans qu'on ait le temps de les saisir (away dans Dombey and Son), on les passe (past): le voyageur reprend ses droits, et avec ceux-ci le temps de l'observation.

C'est ce double phénomène de confusion et de fragmentation du paysage qui est évoqué, parfois seulement dans l'un de ses aspects, par la plupart des voyageurs en train. Les descriptions sont souvent hachées, une simple succession de lieux, de choses

24. «Yoho, past hedges, gates, and trees; past cottages and barns, and people going home from work. Yoho, past donkey-chaises drawn aside into the ditch, and empty carts with rampant horses, whipped up at a bound upon the little water-course, and held by struggling carters close to the five-barred gate until the coach had passed the narrow turning in the road. Yoho, by churches dropped down by themselves in quiet nooks, with rustic burial-grounds about them, where the grasses are green and daisies sleep - for it is evening - on the bosoms of the dead. Yoho, past streams in which the cattle cool their feet, and where the rushes grow; past paddock-fences, farms, and rick-yards; past last-year's stacks cut, slice by slice, away, and showing, in the waning light like ruined gables, old and brown. Yoho, down the pebbly dip, and through the merry water-splash, and up at a canter to the level road again. Yoho! Yoho! Charles Dickens, Martin Chuzzlewit, 1843, dans Myron F. Brightfield, Victorian England in its Novels (1840-1870), Los Angeles, University of California Library, 1968, vol. 3, p. 178 (traduction de Françoise du Sorbier, Martin Chuzzlewit, Paris, Gallimard, coll. «Bibliothèque de la Pléiade», 1986, p. 1213). 
proéminentes que l'on a eu le temps d'attraper au vol. On trouve par exemple dans le «vieux journal» d'Elizabeth Thomson King ce récit d'un trajet effectué le 24 mai 1839:

Avant Warrington, la campagne très plate, mais d'apparence prospère et joliment diversifiée par des arbres. Un long arrêt à Warrington, avec bouffées de vapeur sonores; après, paysage ondoyant. Maintenant nous passons à travers une tranchée profonde - maintenant un tunnel! Maintenant des arbres filant à toute vitesse! Une belle région - un canal - à travers une vallée. ${ }^{25}$

Fragmentation jusque dans le style donc, qui tente de s'adapter à la vue offerte par le train.

L'une des conséquences de cette façon d'appréhender le paysage est que ce dernier semble devenir monotone. Dans ce tourbillon où émergent seulement quelques éléments aussitôt oubliés, l'uniformité règne, et lasse le spectateur.

Les heures passées dans un compartiment de train font en général partie des heures les plus calmes et ennuyeuses d'une vie. Collines, vallées, arbres, villes, larges rivières sinueuses, et même quelquefois des aperçus de la mer elle-même, semblent voler devant nous avec une telle rapidité qu'ils aveuglent le regard et lassent l'esprit. La vue la plus belle ne laisse aucune trace dans la mémoire; sa beauté pittoresque, ses retraites ombragées, ses berceaux de verdure, si agréables pour le voyageur qui prend son temps, n'ont pas de charme de la fenêtre d'un wagon. Nous pouvons voler d'un bout du pays à l'autre, sans autres sentiments que ceux de notre profonde lassitude, et de notre gratitude lorsque le voyage s'achève enfin. ${ }^{26}$

On décèle mieux ici les caractéristiques du sentiment de perte, né de la disparition de tous les charmes du paysage traversé à l'occasion d'un voyage en train. Là encore, tout va trop vite pour que les endroits parcourus puissent être d'abord connus, puis appréciés à leur juste valeur. Le trajet en chemin de fer est monotone, et donc stérile: nulle imprégnation par la beauté des lieux n'est possible. Cet effacement de la singularité du paysage et des traces qu'il laissait auparavant dans la mémoire des voyageurs provoque au mieux la tristesse, au pire la colère, des pourfendeurs du chemin de fer. Car, pour ces derniers, voyager ce n'est pas simplement aller d'un point à un autre en ignorant les régions parcourues, c'est bien au contraire découvrir ces dernières, participer à leur individualité et en même temps à ce qu'elles évoquent cette old England qui ne saurait s'exprimer mieux qu'en ses paysages.

\section{Ruskin et le voyageur-colis.}

Nul n'a exprimé ces idées avec plus de force et d'obstination que John Ruskin. Ce grand penseur de la vision, ce défenseur infatigable de la beauté - ou ce qu'il considérait tel - dans la peinture et la nature, n'a jamais cessé ses invectives contre le train,

25. «Before Warrington the country very flat, but rich-looking and prettily diversified with trees. Long stop at Warrington, with steam puffing loudly; afterwards undulating country. Now we pass through a deep cutting - now a tunnel! Now trees flying past! A pretty country - a canal - across a valley. 》 Elizabeth Thomsonking, Lord Kelvin's Early Home, 1909, dans Humphrey Jennings, op. cit., p. 209.

26. «The hours spent in a railway-carriage are generally among the most tedious and uneventful hours in life. Hills, valleys, trees, cities, broad winding rivers, even at times glimpses of the great sea itself seem to fly past with such rapidity as to dazzle the eye and weary the brain. The loveliest scenery leaves no trace upon the memory; its picturesque beauty, shady nooks, and leafy bowers, so agreeable to the leisurely traveller, have no charm from the window of the railway carriage. We may fly from one end of the land to the other, with no feeling save that of our profound weariness, and gratitude when the journey is over." Mary Ann Hardy, A Hero's Work, London, Hurst and Blackett, 1868, vol. II, p. 67. 
qui agressait précisément ce qui est au cœur de son oeuvre: la vue. Philip Mallett ${ }^{27}$ a rapproché avec pertinence la notion d'aeil protecteur développée par Walter Benjamin dans son essai Sur quelques thèmes baudelairiens ${ }^{28}$, des réactions de Ruskin face à la ville victorienne. L'agression perpétuelle de la ville sur l'homme et son regard impose à ce dernier d'être vigilant à tout moment, et de se protéger sans arrêt des chocs éventuels qui le guettent à chaque coin de rue. De cette façon, l'œil ne peut jamais être pénétré à son insu par des images, des sensations qui toucheraient l'âme en ses couches les plus intimes, et tout ce qui est vu reste au niveau conscient de la mémoire, le plus superficiel de tous. L'aura du monde, pour reprendre un terme de Benjamin, est ainsi perdue, et tout ce qui fait la richesse de l'imagination et la source de la création artistique. Tout ce qui rapproche de Dieu aussi, chez Ruskin du moins. Ces remarques, que Philip Mallett appliquent à la ville, trouvent une pertinence nouvelle lorsqu'on les étend à la question du chemin de fer. Si Ruskin ne décolère pas contre ce dernier, c'est bien parce que sa vitesse est une agression nouvelle imposée à l'œil, une raison neuve pour l'œil de se protéger un peu plus de l'extérieur, et de se fermer à l'imprégnation de la beauté du monde. Le train supprime la relation vivante au paysage, il s'attaque aux conditions mêmes (voyage lent, aventureux en un sens, en contact direct avec les régions traversées) qui permettaient une imprégnation inconsciente du paysage dans l'âme des voyageurs, créant ainsi entre eux un lien fort parce qu'émotionnel. Ecoutons Ruskin dans les Sept Lampes de l'Architecture, après sa fameuse défense des bâtiments historiques:

Le calme même de la nature nous est graduellement arraché: des milliers d'êtres qui autrefois, dans leurs voyages nécessairement prolongés, étaient soumis à l'influence du ciel silencieux et des champs assoupis, d'un effet plus grand qu'on ne le soupçonne ou qu'on ne l'avoue, portent maintenant jusque-là l'incessante fièvre de leur existence. Le long des veines de fer qui sillonnent notre pays, battent les pulsations brûlantes de ses efforts, se faisant d'heure en heure plus brûlantes et plus rapides. Toute la vie se concentre dans ces artères palpitantes au centre des cités, on franchit la campagne comme une mer de verdure sur des ponts étroits et nous sommes sans cesse rejetés en foules plus serrées contre les portes des villes. La seule influence qui puisse, là, remplacer celle des bois et des prés, c'est la force de l'ancienne architecture. ${ }^{29}$

Le rythme endiablé - car il détache de Dieu et de sa création - de la ville et du monde moderne victoriens trouve, selon Ruskin, son expression la plus repoussante dans le train, qui empêche l'influence méconnue et pourtant essentielle des paysages sur l'âme de l'homme, ruinant ce qui fait la richesse de son rapport au monde et à l'art. C'est ainsi qu'il faut comprendre sa fameuse invective dans Modern Painters III, où il compare le voyageur en chemin de fer à un colis. Cette attaque se situe, et ce

27. Philip Mallett, «The city and the self», dans Michael Wheeler (éd.), Ruskin and Environment, Manchester, Manchester University Press, 1995, chap. 2.

28. Walter Benjamin, «Sur quelques thèmes baudelairiens», dans Charles Baudelaire, Jean Lacoste (trad.), Paris, Payot, 1979, p. 147-208.

29. «The very quietness of nature is gradually withdrawn from us; thousands who once in their necessarily prolonged travel were subjected to an influence, from the silent sky and slumbering fields, more effectual than known or confessed, now bear with them even there the ceaseless fever of their life; and along the iron veins that traverse the frame of our country, beat and flow the fiery pulse of its exertion, hotter and faster every hour. All vitality is concentrated through those throbbing arteries into the central cities; the country is passed over like a green sea by narrow bridges, and we are thrown back in continually closer crowds upon the city gates. The only influence which can in any wise there take the place of that of the woods and fields, is the power of ancient Architecture." John Ruskin, The Seven Lamps of Architecture, 1849, dans Works, E.T. Cook, A. Wedderburn (éd.), London, Allen, 1907, vol. 8, p. 246 (trad. de G. Elwall, Les Sept Lampes de l'Architecture, Paris, Les Presses d'Aujourd'hui, 1980, p. 207). 
n'est pas un hasard, dans le chapitre sur la morale du paysage, où Ruskin invite à se réjouir - et à se contenter - de la plus petite nouveauté possible découverte au détour d'un chemin:

Si l'attention est éveillée, et les sentiments adéquats, le virage d'une route de campagne, révélant à ses côtés un cottage que l'on n'avait pas vu auparavant, est tout ce dont on a besoin pour se délasser; si on le passe précipitamment, et qu'on essaye de voir deux cottages d'un coup, c'est déjà trop: ainsi, la façon la plus divertissante de voyager, pour toute personne un peu sensé, est de marcher tranquillement pas plus de dix ou douze miles par jour; et tout voyage devient ennuyeux dans la mesure exacte de sa vitesse. Aller par le train n'est pas, à mon sens, voyager du tout; c'est tout au plus «être envoyé» à un endroit, et assez peu différent d'être transformé en colis. [...] Si l'on marche plus de dix ou douze miles, cela gâte trop la journée, et ne laisse pas de temps pour s'arrêter aux bords du ruisseau ou à ses rives ombragées, ou pour tout autre travail à la fin du jour; d'autant que les derniers miles sont susceptibles d'être effectués dans la précipitation, et donc peuvent être considérés comme un terrain perdu. Mais si, avançant avec lenteur, on approche après quelques jours un paysage encore plus intéressant, chaque yard de ce terrain changeant devient précieux et piquant; et l'accroissement continuel de l'espoir et de la beauté environnante, procure à tout esprit sain l'un des plaisirs les plus délicats qui soient; on acquiert aussi un savoir réel de tout ce que l'on cherchait à connaître en voyageant; enfin, on répand ainsi sur tous les lieux un certain sublime, atteint par la sensation juste des espaces qui les séparent. Un homme qui aime vraiment voyager consentirait aussi peu à tasser une journée si plaisante en une heure de train, qu'un gastronome à concentrer, si c'était possible, son dîner en une pilule. ${ }^{30}$

Là encore la critique porte sur la monotonie du voyage en train (dull) et sur la perte d'initiative du passager: il n'est plus sujet actif du voyage, mais un pur objet qu'on envoie d'un endroit à un autre. Impossible dans ces conditions d'avoir une connaissance réelle des régions que l'on traverse, alors qu'une approche plus lente aurait permis de replacer chaque lieu dans son rapport géographique et esthétique avec le reste du pays. Le voyageur est appauvri, car il est devenu insensible à la beauté de ce qu'il traverse; et dans cette perte de son rapport intime au monde (à l'Angleterre même, pour être plus précis), il est privé d'une part de son humanité. D'où l'image du colis:

Tous les voyages en chemin de fer s'adressent aux gens qui, étant pressés, sont par suite, pour un espace de temps donné, malheureux. Nul ne voyagerait ainsi s'il pouvait faire autrement, - s'il pouvait cheminer à loisir par monts et par vaux, entre des haies, au lieu de franchir des tunnels et de suivre des talus: ceux du moins qui y auraient recours n'ont pas le

30. "If the attention is awake, and the feelings in proper train, a turn of a country road, with a cottage beside it, which we have not seen before, is as much as we need for refreshment; if we hurry past it, and take two cottages at a time, it is already too much: hence, to any person who has all his senses about him, a quiet walk along not more than ten or twelve miles of road a day, is the most amusing of all travelling; and all travelling becomes dull in exact proportion to its rapidity. Going by railroad I do not consider as travelling at all; it is merely "being sent" to a place, and very little different from becoming a parcel. [...] If we walk more than ten or twelve miles, it breaks up the day too much; leaving no time for stopping at the stream sides or shady banks, or for any work at the end [p. 371:] of the day; besides that the last few miles are apt to be done in a hurry, and may then be considered as lost ground. But if, advancing thus slowly, after some days we approach any more interesting scenery, every yard of the changeful ground becomes precious and piquant; and the continual increase of hope, and of surrounding beauty, affords one of the most exquisite enjoyments possible to the healthy mind; besides that real knowledge is acquired of whatever it is the object of travelling to learn, and a certain sublimity given to all places, so attained, by the true sense of the spaces of earth that separate them. A man who really loves travelling would as soon consent to pack a day of such happiness into an hour of railroad, as one who loved eating would agree, if it were possible, to concentrate his dinner into a pill. " John Ruskin, Modern Painters III, 1856, dans E.T. Cook et A. Wedderbum (éd.), Works, London, Allen, 1907, vol. 5, p. 370-371. 
sentiment du beau suffisamment développé qu'il nous faille songer à le consulter à la gare. Le chemin de fer est sous tous les rapports question d'affaire urgente, dont il se faut libérer le plus vite possible. Il transforme le voyageur en colis vivant. Pour un temps donné, le voyageur échange les caractéristiques plus nobles de son humanité contre une puissance planétaire de locomotion. Ne lui demandez pas de rien admirer. ${ }^{31}$

Devenu simple colis d'un voyage monotone, le passager du train est brusquement détaché de l'expérience de la vision picturale du paysage qui était la sienne avant la révolution ferroviaire: il n'a plus la liberté de composition de la scène vue, dont le détail s'efface dans la vitesse de la machine, et plus de participation émotionnelle aux régions traversées, qui perdent de leur individualité dans une vision tourbillonnante et confuse. Le point de vue stable et unique s'échappe lui-même. Quelles réponses apporter à ce trauma du regard, qui est aussi celui d'une identité ?

\section{Des guides à la recherche d'un point de vue.}

Il n'a pas fallu attendre longtemps après l'ouverture des premières lignes pour que des guides de voyage spécifiquement conçus pour le train apparaissent, parfois à l'instigation des compagnies elles-mêmes, trop heureuses de faire échec à leurs détracteurs par des ouvrages tenant parfois plus du dithyrambe que de l'observation neutre. L'une des séries les plus populaires, et les plus précoces, est celle publiée par Arthur Freeling dès 1835, débutant comme il se doit avec la ligne Manchester-Liverpool, et se poursuivant par les descriptions d'autres liaisons ferroviaires comme celle de Londres à Birmingham. Les ouvrages de Freeling sont d'autant plus intéressants qu'ils reçoivent le patronage des directeurs de compagnies, à qui il dédie ses guides ${ }^{32}$. Chaque page de ces derniers se trouve encadrée par deux colonnes, celle de gauche indiquant les distances à partir de la gare considérée comme celle de départ, celle de droite celles de la gare d'arrivée. Au milieu, les objets d'intérêt que le voyageur ne doit pas manquer, et dont un aperçu historique, géographique ou anecdotique est donné. La dernière partie du guide contient enfin des développements spécifiques aux grandes villes concernées par le chemin de fer en question (Birmingham, Manchester, Liverpool, etc.).

Dans la Préface de son ouvrage sur la ligne Londres-Birmingham, Freeling précise clairement les buts de son entreprise:

En proposant le London and Birmingham Railway Companion au Public, je me permets de préciser que j'ai essayé de présenter les informations de telle manière que l'on puisse facilement se reporter à chaque objet digne d'attention lorsque le Train le dépasse, chacun étant évoqué au niveau de la borne kilométrique la plus proche; de ce fait, malgré la vitesse stupéfiante du déplacement, le voyageur peut, en regardant l'ouvrage juste avant d'arriver a une borne précise, obtenir facilement la vue qu'il désire. Mon objectif a été, en fait, de pro-

31. «The whole system of railroad is addressed to people who, being in a hurry, are therefore, for the time being, miserable. No one would travel in that manner who could help it - who had time to go leisurely over hills and between hedges, instead of through tunnels and between banks; at least, those who would, have no sense of beauty so acute as that we need consult it at the station. The railroad is in all its relations a matter of earnest business, to be got through as soon as possible. It transmutes a man from a traveller into a living parcel. For the time he has parted with the nobler characteristics of his humanity for the sake of a planetary power of locomotion. Do not ask him to admire anything. " John Ruskin, The Seven Lamps of Architecture, 1849, dans E.T. Cook et A. Wedderburn (éd.), Works, London, Allen, 1907, vol. 8, p. 159 (traduction de G. Elwall, op. cit., p. 126).

32. «to George Carr Glyn, Chairman, and to the directors of the London and Birmingham Railway Company, this work is (by permission) most respectfully dedicated by their obedient servant. "Arthur Freeling, The London and Birmingham Railway Companion, London, Whittaker, 1838, p. III. 
duire réellement un Manuel de voyage en train, de telle manière que l'on puisse passer le temps de façon plaisante et instructive. ${ }^{33}$

On sent en quelque sorte sourdre au loin l'angoisse de monotonie dont on a relevé la source auparavant: le guide doit permettre, en encourageant une pratique nouvelle (lire à l'avance les informations données afin de ne rien manquer sur la route), de parer à l'obstacle de la vitesse et d'une vision parcellaire, pour redonner au voyage de son intérêt et de son plaisir. Cette allusion à l'apprentissage du regard se retrouve également dans les guides Drake, qui précisent bien que «le voyageur qui souhaite profiter des vues passagères du train, doit se souvenir de sa vitesse de déplacement, et se préparer à voir "tout ce qui peut être vu", ou les objets les plus importants seront passés devant la fenêtre de son confortable wagon à fauteuils, avant qu'on ait pu l'avertir de leur présence. " ${ }^{34}$

Pour aider à cette pratique nouvelle, les auteurs opèrent donc une sélection des objets décrits, en fonction de leur importance pour le passager. Ce choix lui-même est une bonne indication des dispositions nouvelles du regard emporté par le train, et des tentatives des guides pour recréer un point de vue stable. On peut distinguer ainsi trois catégories d'objets dignes d'attention tels que les conçoivent Freeling et ses confrères.

Il y a d'abord la ligne de chemin de fer elle-même: Freeling ne cesse de faire allusion à l'ampleur des travaux effectués, notant chaque talus, tunnel ou viaduc, et précisant alors les tonnes de matériaux déplacés, la taille de ces réalisations, et tous les détails techniques que ses liens avec les propriétaires de la ligne lui permettent d'obtenir (il reproche d'ailleurs à James Cornish, qui a publié un guide concurrent à son Grand Junction Railway Guide, ses erreurs sur ces points). Voici par exemple le type de précisions que l'on trouve à chaque page des guides de Freeling:

Le talus de la Sankey, sur lequel se trouve le voyageur depuis qu'il a passé la borne des 13,5 miles, est le plus lourd de la ligne, atteignant à certains endroits 70 pieds de hauts au-dessus du canal. Le viaduc est construit sur pilotis; la route fait 25 pieds de large, et est soutenue par neuf arches d'une portée de 50 pieds ; l'ouvrage a coûté 45000 livres anglaises. ${ }^{35}$

Ces indications, qui peuvent paraître fastidieuses aujourd'hui, témoignent de la fierté et de l'étonnement des contemporains devant les ouvrages titanesques des ingénieurs ferroviaires, une attitude tellement partagée qu'on la retrouve même chez certains détracteurs du chemin de fer. Quoiqu'il en soit, il convient de noter dès maintenant que ces informations ne peuvent que rarement se référer à la vision offerte par le train à ses passagers: à moins de se trouver à l'extérieur - ce qui était certes le

33. «In presenting the London and Birmingham Railway Companion to the Public, I may be allowed to say, I have endeavoured to arrange the information so that every object worthy of notice may be easily referred to as the Trains pass along, reference being made to each from the mile-post nearest to it; therefore, notwithstanding the amazing rapidity of transit, the traveller may, by looking to the work a little before he arrives at a particular post, easily obtain the desired view. It has, in fact, been my object to produce really a Railway Companion, by whose means the time may be passed pleasantly and instructively. " Arthur Freeling, The London and Birmingham Railway Companion, London, Whittaker, 1838, p. V.

34. "The traveller who would enjoy glimpses of railroads-views, must bear in mind the velocity of his conveyance, and prepare to "see whatever can be seen", or the most important objects will have glanced by the windows of his comfortable locomotive arm-chair coach, before any second person can warn him of their presence." Drake's Road Book to the Grand Junction Railway, Birmingham, James Drake, n.d., p. 50.

35. «The Sankey Embankment, over which the traveller has been passing since he left the post marked 13,5 miles, is the heaviest on the line, being, in some places, 70 feet above the level of the canal. The viaduct is built on piles; the road is 25 feet wide, and is supported by 9 arches of 50 feet span; the work cost 145,000 .» Arthur Freeling, Freeling's Grand Junction Railway Companion to Liverpool, Manchester, and Birmingham, London, Whittaker, 1838, p. 25. 
cas des premiers voyageurs, puis des deuxième ou troisième classes, mais le guide ne s'adressait pas d'abord à ces catégories de personnes - les passagers ne pouvaient voir correctement le trajet de la ligne elle-même, ses viaducs, remblais, etc. Loin de se placer du point de vue du voyageur, Freeling lui en substitue un autre, fixe et extérieur, qui supplée à l'aveuglement du passager. Le problème de la vision à partir du train est ainsi contourné.

Une deuxième catégorie d'éléments notables dans ces guides correspond aux objets qui sont suffisamment proéminents pour être visibles du train et possèdent un intérêt historique. Aussi Freeling évoque-t-il essentiellement villages, églises et grandes demeures, ce qui lui permet d'en brosser un rapide historique et d'en évoquer la condition actuelle (nombres d'habitants dans le village, jours de marché, architecture de l'église, propriétaires actuels de la demeure, etc.). C'est en fonction de cette visibilité et de cette historicité que Freeling sélectionne donc ce que le voyageur ne doit pas manquer d'apercevoir. Sans le dire explicitement, il s'adapte ainsi aux conditions nouvelles du train: il faut trier par avance ce qui doit être vu, car le passager n'est plus en mesure de le faire par lui-même. Le paysage se réduit à quelques éléments éparpillés, dont les espaces intermédiaires semblent vides de sens et d'intérêt. Ainsi, sur la ligne Liverpool-Manchester, après Broad Green Station, Freeling précise-t-il:

La vue ici est étendue et extrêmement pittoresque, et présente plusieurs objets propres à exciter la curiosité du voyageur. Le petit village de Broad Green est sur sa droite - c'est son nom qu'on a donné au talus qui s'étend à travers la vallée sur presque deux miles, varie en hauteur de 15 à 50 pieds, et atteint à certains endroits cent quarante cinq pieds de large à la base. Les divers talus de cette ligne ont été formés par les matériaux dégagés des excavations; $s$ 'il n'avait cette information, le lecteur pourrait se demander où les matériaux nécessaires à des travaux aussi gigantesques pourraient être obtenus. Sur la droite, à une distance considérable, on peut voir Childwell, le siège du Marquis de Salisbury, avec son ancienne église; l'abbaye de Childwell, Roby et Woolton avec ses moulins; et sur une butte élevée, on peut distinguer Halton Castle. Sur la gauche, la vue est encore plus riche et diverse. Au loin se trouvent le Parc et le Manoir de Knowsley, le siège et les terres du Comte de Derby. À la droite de cet endroit richement boisé s'étend la ville de Prescot, dont on aperçoit le clocher de l'église, et à l'exact opposé des wagons se trouve la tour de l'église de Huyton, laquelle, bien que fort éloignée du chemin de fer, semble placée sur ses abords immédiats. ${ }^{36}$

On le voit, il s'agit ici d'aller à l'essentiel: les domaines principaux avec leurs propriétaires, les villages qu'on devine par leurs églises, l'organisation globale du paysage alentours. Aucune attention au détail, bien entendu, la vitesse ne le permet pas. Tout au plus tente-t-on de préserver un point de vue unique, au prix de la précision.

36. «The view here is extensive and exceedingly picturesque, and presents several objects which may excite the enquiry of the traveller. The little village of Broad Green is on his right, - from this the embankment is named, which extends across the valley for nearly two miles, and varies in height from fifteen to fifty feet, and is in some parts, one hundred and forty five feet wide at its base. The various embankments on this line were formed by the materials removed from the excavations; but for this consideration, the reader would wonder where materials for such immense works could have been found. On the right, and at considerable distance, may be seen Childwell, the seat of the Marquis of Salisbury, with its ancient church; Childwell Abbey, Roby, and Woolton with its mills; and on a lofty eminence Halton Castle may be observed. To the left, the scene is even more rich and diversified. In the distance is Knowsley Park and Hall, the seat and grounds of the Earl of Derby. To the right of this richly wooded spot is the town of Prescot, in which may be distinguished the steeple of its church, and exactly opposite to the carriages is the tower of Huyton Church, which, though at some distance from the rail-road, appears from this situation to be [p. $7:$ ] placed immediately thereon." Arthur Freeling, Lacey's Railway Companion and Liverpool and Manchester Guide: Describing all the Scenery on, and contigious [sic] to the rail-way; And pointing out to the visitor at both places all that is interesting and necessary for Business and Pleasure, Liverpool, Henry Lacey, 1835 , p. 6-7. 
Plus intéressant encore, ce qui pourrait constituer une troisième catégorie, et qui se rapproche en fait de la précédente: les paysages «naturels». Lorsque l'auteur décrit une vue, il le fait toujours d'une hauteur, en général à partir d'un talus. Il ne présente le paysage qu'en termes très généraux, ce qui le rend difficilement distinct des paysages précédemment traversés et décrits. On trouve ainsi sur la Grand Junction Line des descriptions de ce type:

Nous approchons maintenant des frontières du Staffordshire, qui sont à moins d'un demimile sur la gauche, et restent à cette distance pour les deux miles suivants, jusqu'à ce que l'on pénètre dans ce comté. De cet endroit, regardant sur notre gauche, se trouve une autre vue digne du pinceau de Claude - collines et vallées, bois et villages, recouvrant un comté dont la surface n'est dépassée en richesse que par les trésors minéraux contenus en son sein. ${ }^{37}$

Ou encore, en passant plus loin sur un remblais au niveau du village de Rickersford:

On a là une vue étendue sur ce beau comté, parsemé des sièges de la noblesse et de la «gentry». Le Val de Shugborough retient l'œil dans son errance à travers une région regorgeant de tous les types de beauté; la Trent argentée et la Sow sinueuse irriguent cette vallée fertile, et les collines d'Acton, se dressant dans une calme majesté, à une distance de plusieurs miles, bornent la vue. ${ }^{38}$

Toujours un grand degré de généralité dans les descriptions qui sont faites, donc, et qui se situent volontairement dans la tradition pittoresque, avec laquelle elles partagent en effet l'insistance sur la structure du paysage (la seule chose que l'œil soit capable d'apercevoir) telle qu'on la découvre d'un point de vue élevé et unique (de cet endroit). Sans parler des éléments mêmes qui constituent ces vues, bien ancrées aussi dans l'imaginaire du paysage anglais «typique», avec leur variété (bois, vallées, collines), la douceur de leurs rivières et la paix qui s'en dégage. On assiste bien ici à une tentative pour masquer le traumatisme du regard produit par le train en recréant artificiellement les conditions dans lesquelles le monde était vu auparavant. Ce désir de compensation est poussé parfois à l'extrême: il arrive ainsi que l'on conseille explicitement un point de vue extérieur au train pour admirer les environs. L'exigence de point de vue stable déniée par le train aboutit ainsi à une sorte de schizophrénie du regard.

Voilà ce que l'on observe du haut du talus d'Arpley:

En regardant vers l'ouest, les hautes cheminées de Runcorn et le château d'Halton se distinguent clairement. La vue à partir des ruines de cette ancienne forteresse, détruite dans les guerres civiles, est très étendue; nous n'avons pas la place ici pour la décrire, mais si nos lecteurs séjournent pour quelque temps à Liverpool, nous ne saurions trop leur conseiller de prendre le bateau à vapeur pour Runcorn, leur assurant une réelle satisfaction à la visite du château et de ses environs. ${ }^{39}$

37. «We are now approaching the borders of Staffordshire, which are but half a mile to the left, and continue about that distance for the next two miles, when we enter that county. From this spot, looking to the left, is another scene worthy of the pencil of a Claude - hill and valley, wood and village, covering a county, the surface of which is only surpassed in riches by the mineral treasures contained in its bosom. » Arthur Freeling, Freeling's Grand Junction Railway Companion to Liverpool, Manchester, and Birmingham, London, Whittaker, 1838, p. 65.

38. «Here is a very extensive prospect of this beautiful county, studded with the seats of nobility and gentry. The Vale of Shugborough detains the eye as it wanders across a country abounding with every variety of beauty; the silver Trent and meandering Sow, water this fertile valley, and the Acton hills, rising in calm majesty, at a distance of many [p. 103:] miles, bound the view. » Arthur Freeling, Freeling's Grand Junction Railway Companion to Liverpool, Manchester, and Birmingham, London, Whittaker, 1838, p. $102-103$.

39. «Looking to the westward, the high chimneys at Runcom, and Halton Castle may be plainly seen. The view from the ruins of this ancient fortress, which was demolished in the civil wars, is very extensive; 


\section{Peinture et paysages en train}

Que le train ait remis en question l'un des fondements de la vision du monde qui l'a précédé, en bouleversant la stabilité du point de vue, est une donnée majeure qui trouve bien entendu des échos dans la peinture de paysage. Puisque c'est avant tout d'une vue picturale du monde qu'il s'agissait, il est naturel de chercher dans la peinture des réponses à ces changements. Or, là comme ailleurs, la confusion règne, et les solutions proposées tendent à contourner le problème plutôt qu'à l'aborder de front.

En effet, si les représentations du chemin de fer sont relativement nombreuses dans les premières années de son apparition, très rares sont les œuvres donnant à voir un paysage aperçu du train. Le point de vue est systématiquement hors de la machine, sur une éminence à part. On le constate aussi bien dans les premières grandes séries d'illustrations de chemin de fer, celles effectuées dès 1831 par Thomas Talbot Bury pour la ligne Liverpool-Manchester et les deux célèbres «long prints» de Isaac Shaw (Travelling on the Manchester and Liverpool Railway en 1831 aussi), que dans les représentations de John Cooke Bourne (Drawings of the London and Birmingham Railway en 1839). À chaque fois, c'est le train qui est représenté, non une vue à partir de lui. Bien entendu, il est possible d'objecter à cela que c'est le train lui-même qui était somme toute la nouveauté, et qu'il était donc logique qu'il soit le centre des représentations. Il n'en reste pas moins que les titres de certains recueils d'illustrations suggèrent eux-mêmes une volonté d'attention portée à l'environnement de la ligne de chemin de fer (ainsi de l'autre recueil d'Isaac Shaw, toujours en 1831, Views of the most interesting Scenery on the [...] Liverpool and Manchester Railway), et qu'il est surprenant qu'aucune représentation ne soit jamais faite à partir du train lui-même lorsqu'il est en mouvement. De même, dans ses Rides on Railways ${ }^{40}$, Samuel Sidney parsème son ouvrage d'illustrations, toutes prises d'un point de vue extérieur et englobant le train. C'est là, sans doute, un signe de l'embarras des peintres devant les perceptions nouvelles apportées par le chemin de fer. Il n'y a pas d'équivalent chez eux des tentatives littéraires de reproduire le regard nouveau que le train permet de porter sur les choses. Ce défi qu'était la représentation de la vitesse en peinture, et l'impossibilité pour la plupart de le relever, se retrouve dans la représentation des trains euxmêmes, qui, comme l'a bien souligné Michael Freeman, tiennent presque de la nature morte, tant l'engin est rendu de façon statique. Ce qui explique que l'intérêt se soit porté le plus souvent sur les infrastructures spectaculaires nées de l'imagination et du travail des ingénieurs:

Alors que J.C. Bourne produisait ses dessins du Great Western Railway au milieu des années 1840, une iconographie caractéristique du chemin de fer était apparue, centrée sur ses structures statiques par opposition aux trains qui leur passaient dessus. À bien des égards, Bourne a fourni au public des voyageurs des images qu'ils ne pouvaient voir du train luimême. La vue de la fenêtre d'un wagon pouvait certes offrir aux passagers des panoramas inédits auparavant [...], mais la plupart ne pouvaient apprécier les traits précis des structures

we have not space to describe it, but if our readers are about to sojourn for any time at Liverpool, we would recommend them to take the steam boat to Runcom, and promise them much gratification in a visit to the castle and neighbourhood. » Arthur Freeling, ibid., p. 38.

40. Samuel Sidney, Rides on Railways, London, William S. Orr \& Co., 1851. 
de la ligne au cours de leur voyage. La vitesse du train provoquait la fusion de ces particularités - elles se perdaient dans la vélocité du «vol» ferroviaire. ${ }^{41}$

Il est une exception majeure, bien entendu, mais elle de celles qui confirment la règle: le fameux Rain, Steam and Speed de Turner, exposé en 1844 à la Royal Academy ${ }^{42}$. On a là sans doute la première tentative - réussie! - de répondre au défi posé par le chemin de fer au regard de l'artiste. Il fallait le génie d'un Turner pour qu'un peintre ose affronter cette aporie technique que semble être l'absence de point de vue stable et le rendu de la vitesse. Les commentateurs de l'époque ne s'y sont pas trompés: le Times écrit ainsi, le 8 mai 1844:

Son Rain, Steam, and Speed présente le Great Western dans une perspective très inattendue, instantanée [...]. Nous laissons les détracteurs et admirateurs de Turner décider entre eux si ses peintures sont des irréalités éblouissantes, ou des réalités saisies d'un coup d'œil. ${ }^{43}$

Quant à la critique de Thackeray, elle est restée célèbre:

Quant à M. Turner, il a surpassé tous les prodiges précédents. Il a réalisé une peinture avec une pluie réelle, derrière laquelle se trouve un soleil réel, et l'on attend un arc-en-ciel à chaque instant. Pendant ce temps, un train surgit vers vous, se déplaçant réellement à la vitesse de 50 miles par heure. ${ }^{44}$

Et l'on rapporte toujours les propos pourtant douteux de Lady Simon qui, ayant voyagé avec Turner sur la Great Western Line, aurait reconnu dans son tableau ce qu'il lui avait fait observer par la fenêtre de leur wagon. Néanmoins, dans cet exemple encore, si exceptionnel soit-il, l'accent est mis sur le train lui-même, non sur ce que l'on voit à partir de lui, d'autant que le point de vue d'où est tiré le tableau reste un mystère (le train représenté n'est que sur une ligne, Turner ne pouvait donc être dans un train allant dans la direction opposée).

On trouve enfin d'autres paysages apparaissant de manière incidente dans des tableaux portant sur des scènes de vie dans les trains. Ainsi de To Brighton and Back for 3s $6 d$, de Charles Rossiter (n.d.), ou du diptyque First Class et Second Class d'Abraham Solomon (1854). Ces vues sont par définition marginales, et se situent le plus souvent dans un coin du tableau. Elles ne tentent en aucun cas de reproduire la sensation de vitesse et de perturbation du regard provoquée par le voyage. Il s'agit là de représentations traditionnelles qui ignorent le défi du mouvement en peinture.

On est donc bien face à un problème dont personne ne semble avoir la solution: comment donner à voir ce qui se déroule de la fenêtre d'un train? L'apparente impos-

41. «By the time that J.C. Bourne produced his drawings of the Great Western Railway in the mid 1840s, a distinctive iconography of the railway had emerged, centred on the railway's static structures as distinct from the trains that ran upon it. In many respects, Bourne provided the travelling public with pictures that they could not have seen from the trains themselves. The view from the carriage window may well have offered passengers previously unobserved vistas [...], but many could not have appreciated the precise features of the structures of the lines as they travelled. The speed of the train caused features to coalesce - they became lost in the velocity of railway "flight". " Michael Freeman, Railways and the Victorian Imagination, New Haven/London, Yale University Press, 1999, p. 230.

42. Voir John Gage, Turner: Rain, Steam and Speed, London, Penguin, 1972.

43. «His "Rain, Steam, and Speed" shows the Great Western in a very sudden perspective [...]. Whether Turner's pictures are dazzling unrealities, or whether they are realities seized upon at a moment's glance, we leave his detractors and admirers to settle between them." The Times, 8 mai 1844, cite dans John Gage, op. cit., p. 13-14.

44. «As for Mr Turner, he has out-prodiged all former prodigies. He has made a picture with real rain, behind which is real sunshine, and you expect a rainbow every minute. Meanwhile there comes a train down upon you, really moving at the rate of fifty miles an hour." William M. Thackeray, Fraser's Magazine, juin 1844, cité dans John Gage, op. cit., p. 14. 
sibilité de cette représentation, et les limites de cette vision neuve par rapport à celle qui la précédait, imposent, lorsqu'elles sont ressenties comme une perte, une forme de compensation au regard amoindri. C'est à cela que tentent de répondre partiellement les guides de voyage.

\section{Des guides pour compléter le regard}

La nécessité de pallier les déficiences du regard provoquées par la vitesse se ressent dès les titres des guides de Freeling, celui de la ligne Manchester-Liverpool ayant ainsi pour objet de «décrire tous les paysages sur et contigus au chemin de fer; d'attirer l'attention du visiteur dans ces deux cas sur tout ce qui est intéressant et nécessaire aux affaires et au plaisir» ${ }^{45}$.

Par ailleurs, il prévient dès la préface de son guide sur la Grand Junction Line:

Dans certains cas, les manoirs de la noblesse que j'évoque ne peuvent être vus lors du passage du voyageur, la pente d'une tranchée, ou une colline, s'interposant de temps en temps; mais j'ai pensé que ce travail serait incomplet si, pour cette raison, des éléments importants devaient passer inaperçus; car, sans eux, aucune idée précise de l'activité du pays ne pourrait être obtenue. ${ }^{46}$

Le chemin de fer ne donnant qu'une vision partielle du paysage, il importe de la compléter pour qu'une idée juste des endroits traversés s'inscrive dans l'esprit des voyageurs. C'est une préoccupation que l'on retrouve chez d'autres auteurs de guides ferroviaires. Leurs titres aussi reprennent la même volonté d'exhaustivité qu'on a rencontrée chez Freeling, comme on le voit chez James Wyld par exemple:

Le Guide de la Ligne Londres-Birmingham, et son Manuel:

Contenant

Une Description Minutieuse du Chemin de fer et de tout Objet digne d'Attention;

Un compte rendu topographique et historique des villes, villages et demeures de la noblesse et des gentlemen,

À une distance de 10 miles de la ligne. ${ }^{47}$

Dans ce dernier guide, l'auteur précise dans sa préface les moyens de cette exhaustivité:

On ne doit pas croire que le présent ouvrage prétend retenir l'attention par l'originalité du contenu qu'il compile, mais plutôt par la parfaite adaptation des documents topographiques et historiques existants, corrigés et augmentés par l'observation personnelle des différentes choses et localités. L'auteur pense avoir obtenu celle-ci (dans la mesure de ses moyens) par une randonnée de Londres à Birmingham. Dans ce voyage, la ligne de chemin de fer a été strictement et scrupuleusement parcourue, de telle sorte que l'aspect de cette région des

45. Arthur Freeling, Lacey's Railway Companion and Liverpool and Manchester Guide: Describing all the Scenery on, and contigious [sic] to the rail-way; And pointing out to the visitor at both places all that is interesting and necessary for Business and Pleasure, Liverpool, Henry Lacey, 1835.

46. «In some cases, the noblemen's seats referred to cannot be seen as the traveller passes along, the slope of a cutting, or a rising hill, occasionally intervening; but I have thought the work would be incomplete if, on this account, an important object were unnoticed; as, without it, no accurate idea of the occupation of the country could be obtained. " Arthur Freeling, The London and Birmingham Railway Companion, London, Whittaker, 1838 , p. V.

47. The London and Birmingham Railway Guide, And Birmingham and London Railway Companion: Containing A Minute Description of the Railroad, and every Object worthy of Notice; An antiquarian and topographical account of the towns, villages, noblemen and gentlemen's seats, Within ten miles of the railroad. James W. Wyld, London, 1838. 
deux côtés du voyageur ait pu être relevé, et que les villes, villages et objets dont il est question dans les pages suivantes aient pu être visités et examinés.

L'aspect que certaines scènes et objets présentent lorsqu'ils sont vus du train a été décrit, afin qu'ils puissent être facilement reconnus; et l'on ne mentionne que ce qui peut être vu par le voyageur lors de son déplacement, ou accessible à partir des différentes gares de la ligne. ${ }^{48}$

Même remarque, enfin, dans le guide de Francis Coghlan:

\section{AVIS.}

L'auteur du Iron Road Book ayant marché le long de toute la ligne de chemin de fer ouverte aujourd'hui entre Londres et Birmingham, au prix de lourds efforts personnels (car c'est là le seul moyen de donner une description correcte de la ligne), pense qu'il est nécessaire de préciser qu'un grand nombre de lieux décrits ici ne peuvent être vus du chemin de fer; on a remédié en revanche à la nudité des environs immédiats de la ligne en donnant les descriptions des villes et villages les plus intéressants de chaque côté, même si on ne peut les distinguer des wagons. ${ }^{49}$

Un déplacement en chemin de fer ne peut se concevoir ainsi qu'à condition d'avoir été précédé d'un voyage à pied qui donne seul l'occasion de voir et de connaître correctement les lieux traversés. C'est par ce complément pédestre seul que la vue à partir du train peut être réhabilitée, quitte à n'offrir au regard parfois que des objets trop lointains pour être visibles, et à appeler l'imagination au secours pour reconstruire l'intégralité de ce qui devrait être vu. Drake n'hésite pas ainsi à décrire le district des Potteries, même s'il admet lui-même qu'aucune des villes qui le composent n'est à portée de vue du chemin de fer ${ }^{30}$.

Et Cornish évoque un autre moyen de cette compensation:

comme cette façon de voyager est trop rapide pour permettre au voyageur d'obtenir plus qu'un coup d'œil momentané, nous nous proposons de pallier ceci autant que possible dans

48. "It must not be supposed, that any claim which the following work may have to notice, is based on the originality of the matter to be found in its compilation, but, rather, on the fitting adaptation of existing topographical and antiquarian documents, emended and enlarged by a personal observation of the several objects and localities. This the author presumes (to the extent of his ability) to have been effected in a pedestrian tour by him from London to Birmingham. In this tour, the line of [p. II:] railroad was strictly and scrupulously travelled, in order that the appearance of the country on either side of the traveller might be noted, and those towns, villages, and objects, discoursed of in the following pages, visited and examined. The appearances which particular scenes and objects present when viewed from the train, have been described, so that they may easily be recognised; and those only are mentioned which may be seen by the traveller on his journey, or approached from the different stations on the railroad." James $W$. Wyld, The London and Birmingham Railway Guide, And Birmingham and London Railway Companion: Containing A Minute Description of the Railroad, and every Object worthy of Notice; An antiquarian and topographical account of the towns, villages, noblemen and gentlemen's seats, Within ten miles of the railroad, London, James Wyld, 1838, p. I-II.

49. «NOTICE. The author of the Iron Road Book having walked the entire line of Railroad now open, between London and Birmingham, at great personal fatigue (as by no other means could a correct description of the line be given), considers it necessary to state that a great number of places described cannot be seen from the line; the bareness of the immediate neighbourhood of the railway has, however, been supplied by giving descriptions of the most interesting towns and villages on either side, although not distinguishable from the carriages. " Francis Coghlan, The Iron Road Book and Railway Companion; Or a journey from London to Birmingham Containing an account of the Towns, Villages, Mansions, \&c. on each side of the line, London, A.H. Baily \& Co., 1838, p. 22.

50. «Bien qu'aucune des villes de ce grandiose district appele les Potteries n'est sur la ligne, ou même visible du train, elles sont si proches qu'il ne serait presque pas convenable de ne pas faire mention de cet imposant voisinage industriel.» «Although none of the towns in the great district called the Potteries, are upon, or even seen from, the line of the route, yet they lie so near, that it would scarcely be well to omit all mention of this great manufacturing [p. 36:] neighbourhood." Drake's Road Book of the Grand Junction Railway, Birmingham, James Drake, n.d., p. 35-36. 
le présent ouvrage, en donnant un bref historique de la région et des villes situées sur la ligne ou dans son voisinage immédiat ${ }^{\mathrm{s}}$.

L'historique des lieux traversés est ainsi explicitement présenté comme la meilleure manière de compenser l'impossibilité de les voir correctement. Comme si la connaissance intellectuelle du lieu devait remplacer, pallier, l'ignorance de son apparence!

Car ce que les auteurs cherchent ici à corriger, c'est bien l'effacement des spécificités de chaque paysage, l'uniformité que le chemin de fer impose aux régions qu'il traverse, faute de laisser le temps au voyageur d'apprécier et de déceler leurs différences réelles. D'où ce cri de James Wyld:

Nous émergeons à nouveau dans la clarté et la beauté du jour. Et quelle vue pour se délecter! Si seulement nous pouvions la contempler ne serait-ce que quelques minutes: - mais non! le monstre au cœur d'acier, pour qui déserts, roches et prés fleuris sont semblables, nous entraîne sans espoir, insensible à la poésie de la Nature, aux pentes ceintes de bois, aux eaux sinueuses, reflétant le paysage et les tourelles grises des églises avec les nuances mêlées de milliers de fleurs sauvages; les rochers surplombants, émaillés d'ajoncs dorés, et parsemés de pins assombris, voltigent devant nous, et disparaissent, comme une scène féerique que l'imagination aurait créé sous les cieux de l'orient, ou quelque rêve matinal de royaumes d'au-delà de ce monde, que seuls l'éveil et la réalité peuvent dissiper et réfuter. ${ }^{52}$

Il est étonnant de trouver dans un guide une telle exclamation, qui semble révéler la vacuité de ses efforts pour compléter le regard. Le voyageur moderne a.perdu la beauté des paysages anglais, devenus de purs rêves exotiques (ne peut-on déjà percevoir ici la peur d'un déracinement?), au profit d'une efficacité accrue des transports, dont la vitesse uniformise les scènes traversées. Cet échec des guides ferroviaires, et les dangers identitaires du train, sont dès lors mis en avant par les plus obstinés de ses détracteurs.

\section{Touristes et voyageurs: le déracinement}

C'est précisément parce que le train ne permet qu'une connaissance superficielle du paysage, qu'une tradition intellectuelle différenciant les touristes des voyageurs s'est mise en place, comme l'a très justement souligné James Buzard ${ }^{53}$ : les premiers n'ont pas d'expérience réelle, concrète, de ce qu'ils visitent et voient, ils ne font que passer devant un lieu, au contraire des voyageurs - à l'origine de cette distinction bien

51. «as the mode of travelling is too rapid to allow the traveller to get more than a momentary glance, we propose to obviate this as far as we can in the present work, by giving a brief history of the country and towns, either on, or in the immediate vicinity of, the line». The Grand Junction, and the Liverpool and Manchester Railway Companion Containing an account of Birmingham, Liverpool, and Manchester, And all the towns on or near the line; Together with every thing worthy of the attention and notice of the traveller on the line; Etc., Birmingham/London/Liverpool, James Cornish, 1837, p. 4.

52. «We have emerged again into the bright and beautiful of day-light. But, what a [p. 53:] scene to revel in! Would that we might gaze but for a few short minutes: - but no! the iron-hearted monster, to whom alike are desert, rock, and flowery mead, drags us helplessly along, unaffected by the poetry of Nature, and the wood-girt slope, the meandering water, mirroring the landscape and grey turret church, with the blent hues of a thousand wild flowers; the beetling rock, enamelled with golden gorse, and studded with the blackened pine, flits past us, and is gone, like a bright fary scene which imagination has created in the climes of orient, or some morning dream of realms beyond this life, which waking reality alone can dissipate and falsify." James W. Wyld, The London and Birmingham Railway Guide, And Birmingham and London Railway Companion: Containing A Minute Description of the Railroad, and every Object worthy of Notice; An antiquarian and topographical account of the towns, villages, noblemen and gentlemen's seats, Within ten miles of the railroad, London, James Wyld, 1838, p. 52-53.

53. James Buzard, The Beaten Track: European Tourism, Literature and the Ways to "Culture" 18001918, Oxford, Clarendon Press, 1993. 
entendu - qui se laissent volontiers imprégner par l'esprit du lieu qu'ils découvrent. Il n'est qu'à remonter à Carlyle pour retrouver l'une des expressions de cette différenciation et de cette critique des «expéditions touristiques qui sont plus aveugles que jamais, et effectuées par la vapeur, sans même la faculté de voir, ni de réfléchir évidemment ${ }^{54}$. On a là aussi la raison première de la fameuse opposition de Wordsworth à la ligne Kendal-Windermere en 1844-1845. L'auteur du Guide to the Lakes a été longtemps moqué pour avoir voulu refuser l'invasion touristique de «son» cher domaine des Lacs - une invasion que la ligne de chemin de fer rendait inévitable -, après avoir fait plus que tout autre pour rendre cette région célèbre. Mais dans son guide même il n'a jamais cessé de plaider pour une connaissance vraie, approfondie, de ce joyau anglais, qu'il a cherché à transmettre par le lyrisme de sa poésie. Son romantisme a ses racines dans cette symbiose avec le paysage des lacs et son désir de partager cette relation émotionnelle et intime avec ceux qui pouvaient la comprendre. Et le guide est resté célèbre aussi pour ses attaques contre les industriels s'installant dans la région et construisant des demeures étrangères dans leur esprit au lieu qu'elles défiguraient dès lors. «Wordsworth défendait un idéal de voyage sincère, indépendant, par opposition au tourisme avili qui trop souvent semblait suivre ses traces et le rendre de plus en plus difficile à atteindre.» ${ }^{55} \mathrm{Qu}$ 'il y ait là une réaction d'intellectuel horrifié de voir la plèbe envahir et déformer les endroits solitaires où il cherchait son inspiration, ne fait aucun doute. Mais la réflexion de Wordsworth, comme celle de Ruskin, s'articule autour de questions bien plus larges qui expliquent à terme leur opposition commune aux chemins de fer et à ce qu'ils impliquent sur la façon de voyager et de voir les paysages.

On comprend ainsi pourquoi les arguments sur la démocratisation du transport les atteignaient somme toute assez peu. Il est indéniable pourtant que les paysages anglais, par la grâce du chemin de fer, se sont soudain ouverts à un public plus vaste et plus hétéroclite qu'avant. Les contemporains eux-mêmes ont voulu voir un signe de cette démocratisation dans la proximité temporelle de ces deux événements clés de leur histoire: l'irruption des chemins de fer commerciaux en 1830 et le Reform Act de 1832. Que les trains se soient bientôt ouverts à tous, est une réalité indéniable que Thomas Cook, qui le premier a songé à organiser des excursions en chemin de fer bon marché, a grandement aidé à établir. En 1850, lors d'une seule semaine de congés, quelque 200000 personnes ont quitté Manchester par des cheap excursion trains. L'impact sur la connaissance du pays est souligné par nombre de commentateurs:

Des hommes qui, il y a quelques années encore, traversaient à peine les limites du comté où ils étaient nés, et connaissaient aussi mal les traits généraux de leur pays de naissance que la topographie de la lune, profitent désormais sans hésiter des moyens de communication qui leur sont disponibles. ${ }^{56}$

54. «touring expeditions which are blinder than ever, and done by steam, without even eyesight, not to say intelligence." Thomas Carlyle, The History of Friedrich II of Prussia, Called Frederick the Great, London, Chapmann and Hall, 1859, vol. II, p. 181 ( $3^{\mathrm{e}} \mathrm{ed}$.).

55. «Wordsworth promoted an ideal of sincere, independent travel against the degraded tourism that so often seemed to follow in its footsteps and to make it increasingly difficult to practice." James Buzard, op. cit., p. 29.

56. «Men who but a few years since scarcely crossed the precincts of the county in which they were born, and knew as little of the general features of the land of their birth as they did the topography of the moon, now unhesitatingly avail themselves of the means of communication that are afforded." F.S. Williams, Our Iron Roads: their history, construction and social influences, London, 1852, p. 285. 
À quoi Wordsworth, Ruskin et leurs disciples répondent que le chemin de fer ne permet en aucune manière une meilleure vue (et donc connaissance) de la terre anglaise, d'autant qu'en transportant des hordes de touristes il détruit les endroits même qu'il veut donner à admirer.

On a prodigieusement vanté les mérites du chemin de fer et du bateau à vapeur, et nous n'avons aucune intention d'en déprécier les avantages. [...] Mais ils ont infligé à notre génération un mal épouvantable: ils ont recouvert l'Europe de Touristes. ${ }^{57}$

Et les attaques ne s'arrêtent pas là: outre ses méfaits sur le voyage lui-même, le chemin de fer semble, selon ses détracteurs, mettre à mal le sens de la communauté, l'attachement au lieu où l'on est né et où l'on vit. En réduisant l'espace, le train détruirait les différences entre les lieux, et nuirait à la compréhension de leur spécificité. En accélérant le temps, il ne donnerait plus les chances d'une connaissance réelle et intime des paysages constitutifs d'une personnalité. C'est le sens de la déploration de Thomas Hughes dès le premier chapitre de Tom Brown's Schooldays:

O jeunesse d'Angleterre! jeunesse d'Angleterre! Toi qui est née dans ces temps précipités du chemin de fer, [...] tu peux traverser quelques milliers de miles pour trois livres dix, en cinq semaines de vacances; pourquoi ne connais-tu pas mieux ton propre lieu de naissance? Tu me sembles tout entière au bout du monde dès que tu sors la tête du joug éducatif, pour les vacances d'été, les grandes vacances, que sais-je encore. [...] Je dis juste que tu ne connais pas tes propres chemins, bois et prés. [...] En mon temps, lorsque nous rentrions encore chez nous par la vieille diligence [...], nous devions inventer nos propres jeux à proximité de la maison, pas plus loin qu'une marche ou qu'une course à cheval. C'est ainsi que nous avons pu connaître les gens de la campagne, et leurs coutumes, leurs chansons et leurs histoires par cœur; et nous allions par les prés, et les bois, et les collines, encore et encore, jusqu'à ce qu'ils soient tous des amis. Nous étions tous des enfants du Berkshire, ou du Gloucestershire, ou du Yorkshire, tandis que tu es une jeunesse cosmopolite, de tous les comtés, et d'aucun pays. ${ }^{58}$

L'ultime reproche fait au train est donc bien celui d'un déracinement. Le chemin de fer ne permet plus d'apprécier à leur juste valeur les paysages de l'Old England, et de se laisser toucher par eux. L'identité anglaise elle-même vacille dans cette ignorance nouvelle.

\section{Conclusion}

Les critiques faites au chemin de fer sur les limites de la vision des paysages qu'il offrait ne peuvent donc se réduire à une simple plainte rétrograde vantant a contrario les bienfaits du voyage en diligence. Il y a certes parfois une part de mauvaise foi

57. «The merits of the railroad and the steamboat have been prodigiously vaunted, and we have no desire to depreciate the advantages of either. $[. .$.$] But they have afflicted our generation with one desperate$ evil; they have covered Europe with Tourists. » «Modern Tourism», Blackwood's Magazine, vol. 64 (aout 1848), p. 185.

58. «O young England! young England! You who are born into these racing railroad times, $[\ldots]$ you can get over a couple of thousand miles of ground for [p. 22:] three pounds ten, in a five weeks' holiday; why don't you know more of your own birthplaces? You're all in the ends of the earth, it seems to me, as soon as you get your necks out of the educational collar, for midsummer holidays, long vacations, or what not. (.... All I say is, you don't know your own lanes and woods and fields. (...] Now, in my time, when we got home by the old coach [...] we had to cut out our own amusements within a walk or ride of home. And so we got to know all the country folk, and their ways and songs and stories by heart; and went over the fields, and woods, and hills, again and again, till we made friends of them all. We were Berkshire, or Gloucestershire, or Yorkshire boys, and you're young cosmopolites, belonging to all counties and no countries. » Thomas Hughes, Tom Brown's Schooldays, London, Penguin, 1997 (1 ${ }^{\text {re }}$ éd. 1857), p. 21-22. 
conservatrice dans cette idéalisation du passé, mais les enjeux posés par le nouveau mode de transport se situent bien au-delà. C'est ce que certains contemporains ne semblent pas avoir compris, d'ailleurs, comme en témoigne cet extrait de The Illustrated London News:

On prétend que l'on ne peut pas voir les paysages à partir du train. Nous le réfutons. Regardez les innombrables et délicieuses vues que l'on entrevoit alors qu'on les traverse rapidement - [...] - qui dit que des centaines de milliers de charmants morceaux de campagne comme ceux-ci ne peuvent être vus aussi bien de derrière une locomotive que de derrière les queues remuantes des chevaux essoufflés? Le train a aussi l'avantage de ne pas vous garder trop longtemps sur chaque perspective - et que vous ne paraissez jamais comme envoûté par une colline particulière, ou un clocher particulier, que vous semblez ne jamais pouvoir laisser derrière vous. Vous voyez, et passez, et voyez à nouveau. ${ }^{59}$

En cherchant à défendre le chemin de fer, Read alimente en fin de compte les arguments de ses adversaires, puisqu'il met en évidence ici la fragmentation du paysage (on ne perçoit, selon ses propres termes, que des «glimpses» et des «country bits»), et la volatilité du regard et de l'attention des voyageurs, lesquels peuvent désormais échapper au charme des endroits qu'ils traversent.

C'est ainsi un véritable trauma du regard que le train a provoqué, mettant à bas toutes les conditions précédentes d'une vue essentiellement picturale du monde. Les guides ont bien cherché (avec un certain succès, puisque certaines de leurs caractéristiques sont encore d'actualité) à répondre à ces perturbations du point de vue en encourageant de nouvelles pratiques, et en compensant la partialité de l'observation par l'intellectualisation du paysage, réduit à ses grandes lignes et à des données historiques, géographiques, ou anecdotiques. Néanmoins, si les critiques sont restées aussi vives, c'est bien parce que la relation même des voyageurs à l'Angleterre, et donc à leur identité, paraissait en jeu: en effet, l'esthétisation progressive des paysages anglais depuis le milieu du XVIIr ${ }^{e}$ siècle avait fini par faire de ceux-ci l'un des éléments clés de la définition de l'Englishness. En bouleversant le rapport au paysage, devenu, de la fenêtre du train, confus, monotone, réduit à quelques éléments détachés de tout contexte, et surtout extérieur à l'expérience concrète du voyageur, le chemin de fer semblait, pour ses détracteurs, mettre en danger le cœur même de l'identité nationale. Il devenait ainsi pour eux l'acteur principal de la déshumanisation à l'œuvre dans le processus d'industrialisation, qui ruinait les attaches communautaires de l'Angleterre, et transformait ses habitants en êtres sans racines et privés de la richesse d'un rapport émotionnel aux paysages qui seul les distinguait de la machine.

UNIVERSITÉ MICHEL DE MONTAIGNE - BORDEAUX III

59. «It is said that you cannot see the country from the rail. We deny it. Look at the multitudinous sweet glimpses we catch as we hurry by - [...] - who says that hundreds of thousands of such charming country bits may not be seen from behind the locomotive as well as from behind the twitching tails of the panting "prads"? And the advantages of the railroad are, that you are not kept too long over any one prospect - that you never appear as if spellbound by a particular hill, or a particular church tower, which you seem never to be able to leave behind you. You see and pass, and see again. " Angus B. Read, «The Railway Note-Book», Illustrated London News, 19 juin 1852, p. 486. 